\title{
Single-stranded DNA plant pathogens in Eilat
}

\author{
Barbara Hohn ${ }^{1}$ and Thomas Hohn ${ }^{1,2, *}$ \\ ${ }^{1}$ Friedrich Miescher Institute for Biomedical Research, Maulbeerstr. 66, Basel CH-4058, Switzerland; \\ ${ }^{2}$ Botanical Institute of the University, Schoenbeinstr. 6, Basel CH-4056, Switzerland (*author for corre- \\ spondence; e-mail hohn@fmi.ch)
}

Received 21 January 2006; accepted in revised form 26 January 2006

Key words: conference, single stranded DNA, Agrobacterium tumefaciens, geminivirus

\begin{abstract}
An international conference on "Inter- and Intracellular Dynamics of ssDNA Plant Pathogens: Implications for Improving Resistance" was sponsored by the United States-Israel Binational Agricultural Research and Deveoplment Fund (BARD) and organized in Eilat, Israel in November 2005. The topic of this meeting was single-stranded plant pathogens, their inter- as well as intra-cellular dynamics and their implications for improving resistance. Most of the talks concentrated on new and very new findings on principles of virus and bacterium-host interactions, studies that no doubt will lead eventually to the establishment of plants resistant to viral and bacterial infections.
\end{abstract}

\section{Introduction}

Around 60 of us lucky ones were selected to assemble in Eilat, Israel, to report on new advances on the interaction of single-stranded (ss)DNA containing plant pathogens with their victim-hosts. This exciting encounter was made possible by BARD, the Israel Binational Agricultural Research and Development Fund that enables research conducted by Israeli researchers in collaboration with American scientists. The conference was organized by an extremely competent team around V. Citovsky (Stony Brook, New York) and Y. Gafni (Volcani Center, Israel). The organizers not only provided exciting scientific challenges, but lured also our senses with an excellent excursion to the wonders of the Eilat Underwater World (Figure 1) and to culinary adventures.

\section{T-DNA, a plant pathogen of bacterial origin}

Research on the intricate interactions of Agrobacterium tumefaciens and plants can be described as attempts to answer a series of questions, including: (1) What happens in the bacterium when it encounters a plant cell? (2) How does the T-DNA, the part of the $\mathrm{Ti}$ plasmid that moves to the plant and becomes integrated into plant genomic DNA (Gelvin, 2003), leave the bacterial cell and how does it enter the plant cell? (3) How do the T-DNA and its accompanying bacterial (virulence proteins) and plant derived proteins move through the cytoplasm and through the nuclear pores? (4) How does the T-DNA integrate? (5) How do the virulence proteins get removed from the T-DNA after they have served their function? and finally, (6) How does a bacterium react once the plant host has been transformed to produce a tumor?

Employing a proteomics approach, E. Ron (Tel Aviv) studied the bacterium's response to plant cells and demonstrated the existence of a plant dependent 'stimulon' consisting of at least 30 novel proteins. In addition, the stimulated bacterium was found to contain a large number of posttranslational protein modifications, some of which were plant induced. Escherichia coli had been 


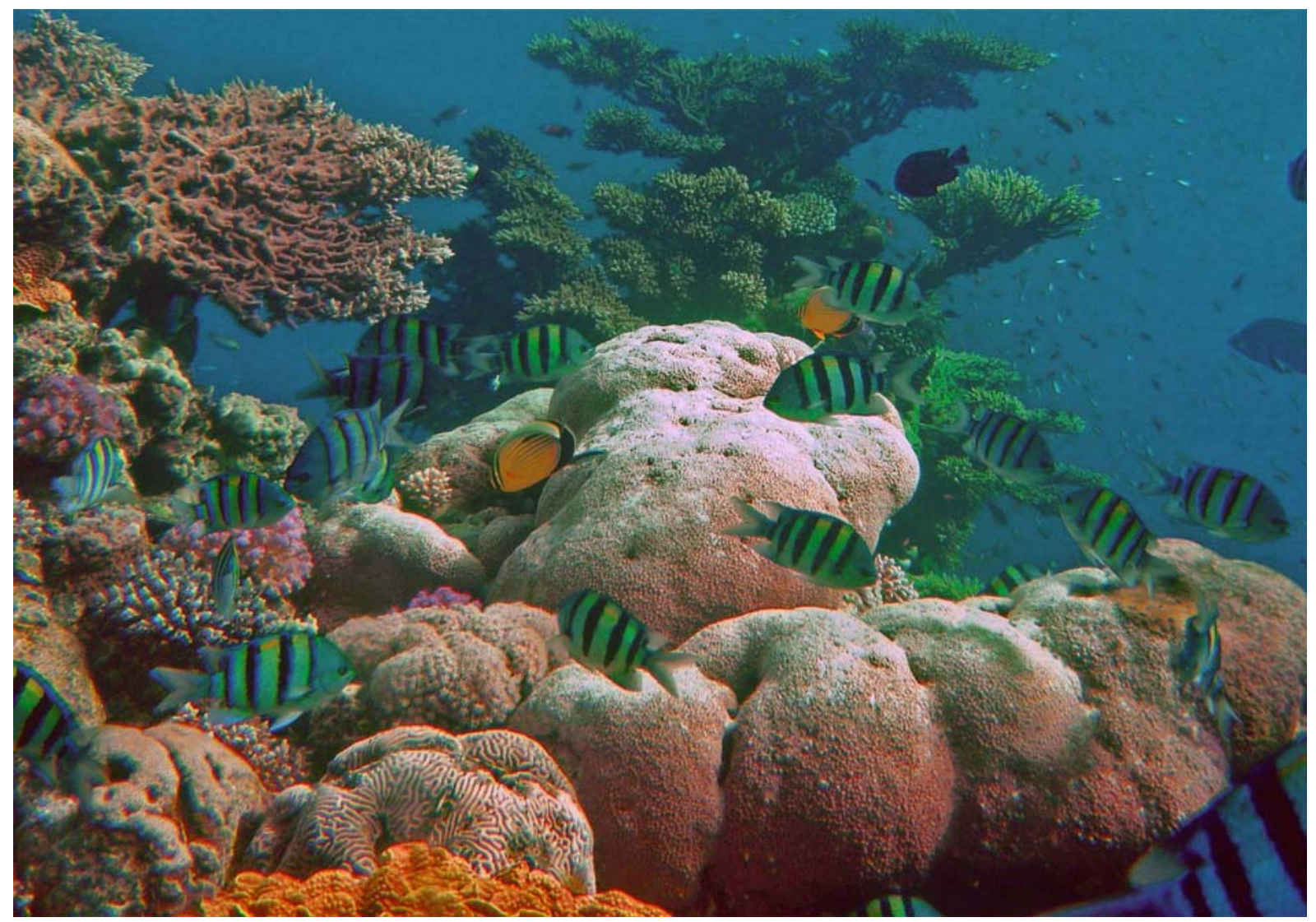

Figure 1. Impressions from an excursion to the Eilat Underwater Observatory (original photo kindly provided by M. Duckely).

shown earlier to possess highly phosphorylated proteins (Rosen et al., 2004).

It is not very clear, how the T-DNA, attached to the virulence protein VirD2, exits the bacterial cell. An ingenious single-stranded (ss)T-DNA/virulence protein immunoprecipitation assay had allowed the identification of sequential contacts of the DNA with the virulence proteins forming the secretory apparatus (Cascales and Christie, 2004). This assay was used by A. Binns (Philadelphia) to show that transfer of plasmid RSF1010 follows a similar translocation pathway. Moreover, reduction of virulence by the conjugal intermediate of RSF1010 was shown to be the result of these molecules blocking the interaction of both the T-strand and VirE2 (see below) with the type IV secretion system. The entry of the T-DNA and its associated proteins into plant cells is a mystery altogether: How could a highly charged molecule such as ssDNA move through the plasma membrane, especially in view of the general acceptance that the ssDNA-binding protein VirE2 becomes translocated to the plant cell separately? A theory based on biophysical experiments that this protein forms a membrane pore through which the T-DNA may enter the eukaryotic cell may solve the puzzle (Dumas et al., 2001). Recent experiments reported by $M$. Duckely (Basel) placed some credence to the model. A VirE2 mutant was isolated, which rendered the protein inactive in membrane association as well as in effecting virulence. Association of VirE2 with its chaperone VirE1 converts VirE2 into a soluble form; in the presence of ssDNA, VirE2, but not VirE1 associates with the DNA, as reported both by M. Duckely (Basel) and M. Elbaum (Rehovot) (Figure 2). It is not known, however, whether VirE1 moves along with VirE2 into the plant cell. Since plants transgenic for VirE2 are independent of VirE1 in their potential to rescue bacteria lacking VirE2 to virulence (Citovsky et al., 1992), the chaperone is most likely only required inside the bacterium.

M. Elbaum also showed most extraordinary EM pictures of a ssDNA/VirE2 protein complex, 


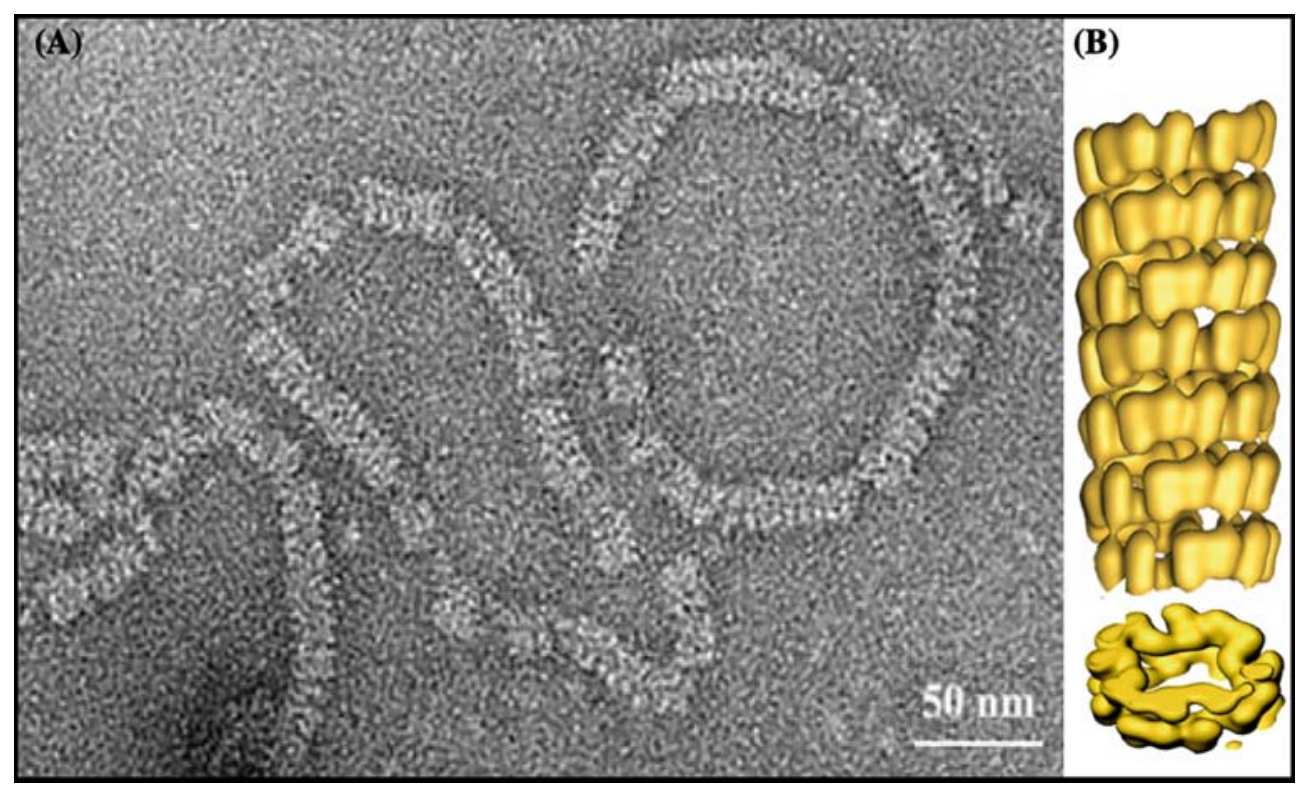

Figure 2. Complex of circular single-stranded DNA with VirE2. (A) Transmission electron micrograph. (B) Model derived from averaging 3000 particle images (after Abu-Arish et al., 2004).

as it moves through a nuclear pore of a plant cell. Not only could he detect the T-complex in flagranti, but he presented the first three-dimensional view of a plant nuclear pore. S. Gelvin (Purdue) isolated several importin $\alpha$ proteins of Arabidopsis thaliana and tested them for interaction with VirD2 and VirE2, both of which were implicated in nuclear transport previously. Yeast two-hybrid-, in vitro protein interaction- and bimolecular fluorescence complementation assays demonstrated that several importin $\alpha$ members interact with VirD2 as well as with VirE2. However, it is not clear whether these interactions are functional, as out of four tested members only the mutant in importin $\alpha 4$ was resistant to transformation by Agrobacterium tumefaciens. Surprisingly, in this mutant, VirD2 was still transported to the nucleus, whereas both in wild type as well as in mutant plant cells VirE2 was found in the cytoplasm. Clearly additional functional assays will be required to resolve these interesting questions. Connected to the problem of nuclear targeting was the report by V. Citovsky (Stony Brook): Agreeing with S. Gelvin on this question, VirE2 by itself is not found in the nucleus, but requires the activity of Vip1, a VirE2 interacting protein, for nuclear targeting. However, the nuclear localization sequences of Vip1 have not been tested. The proteins VirF and VirE3 are also components of the T-complex, at least if strains producing these virulence proteins are used. It is an interesting question how these proteins, as well as VirE2 and Vip1 are removed from the Tcomplex before/during integration of the T-DNA. According to Citovsky, this uncoating occurs by proteosomal degradation of the proteins, through interaction of VirF with the degradation machinery. It is unclear at the moment how the build-up of the proposed multi-component complex is uncoupled from its decomposition; a tight regulation must operate to insure a strict temporal order.

T-DNA integration is normally described as using a pathway of non-homologous end-joining, leading to site-unspecific ligation reactions. At least two events are necessary to accomplish this difficult task: The T-DNA has to gain access to chromatin and enzymes, and additional factors have to be recruited to the site of action. Preliminary data by V. Citovsky point to an association of Vipl with histones (Loyter et al., 2005), but affinity of T-complexes to nucleosomes or bona fide chromatin will have to be tested. Chromatin remodeling activity can be assumed to lead to accessibility of (damaged) chromatin for T-DNA, but the newly identified INO80 protein seems to be specific to homologous recombination 
(B. Hohn, Basel). The chromatin assembly factor CAF-1, however, does seem to play an important role in allowing access to chromatin for both enzymatic activities associated with homologous recombination, as well as for T-DNA and connected integration activities (B. Hohn, Basel, referring to work by S. Toki, Tsukuba). As far as proteins engaged in integration are concerned, analysis of virulence proteins originally was the first choice. However, direct involvement in integration could not be demonstrated for either VirD2 or for VirE2. Instead, the role of VirD2 seems to be rather indirect: VirD2 has been shown to interact with DNA ligase I and to stimulate it by adenylation. Also DNA ligase IV, when overexpressed in Arabidopsis thaliana plants, leads to increased levels of T-DNA integration (B. Hohn, Basel). Other prominent roles in the integration process seem to be reserved to the KU80 homologue of Arabidopsis: in knockout plants the efficiency of T-DNA integration, but not of T-DNA transfer, seems to be greatly reduced. Conversely, plants over-expressing KU80 are resistant to DNA damaging agents and allow an increased rate of T-DNA integration (T. Tzfira, Michigan). This pathway specifically enhances integration by double-stranded versions of the T-DNA, as shown by immunoprecipitation of complexes containing both KU80 and doublestranded (ds)T-DNA (Li et al., 2006).

The last question in the pathway of T-DNA mediated plant transformation, as posed in this meeting, was related to the post-transformation life of Agrobacterium: after the energy-devouring phase of virulence activation and T-DNA transfer and the successful transformation of a plant victim, a deregulation phase seems to be initiated: Transformation, as sensed by the bacterium as plant hormone production, seems to inactivate the virulence of the bacterium (E. Nester, Seattle; see also: Ditt et al., 2005). The concept of posttransformation virulence deregulation remains an exciting one, worth further examination.

\section{Plant viruses containing ssDNA}

\section{Replication}

The second type of ssDNA plant parasites are the geminiviruses and their 'smaller cousins', the nanoviruses. Gemini- and nanoviruses represent all of the known plant virus families that replicate their genome using a DNA polymerase. Nanoviruses are multipartite with eight individual DNAs coding each for one protein and each packaged separately in small icosahedrons. As B. Gronenborn (Gif sur Yvette) pointed out, the key viral protein involved in nanovirus replication is the master replication initiator (m-Rep; Gronenborn, 2004). It recognizes the viral origin of replication on the dsDNA replicative intermediate and cleaves the consensus TAGTATT $\mid$ AC sequence establishing a covalent phosphotyrosyl-AC link. It also sequesters the host DNA polymerase and initiates a rolling circle-type replication. Upon production of a unit length plus-strand ssDNA, a second transesterification reaction releases circularized DNA and $\mathrm{m}$-Rep for reuse. In order to have all components for DNA replication available, nanoviruses and geminiviruses trigger cell cycle progression. For this function nanoviruses encode a separate protein, the cell cycle link protein (CLINK), which binds to retinoblastoma-like protein.

Geminiviruses replicate their genome by a mechanism similar to the one of nanoviruses. However, Rep of begomoviruses, the most studied group of geminiviruses, combines the functions of dsDNA cleavage, DNA polymerase sequestration and the triggering of cell cycle progression. A second early begomovirus protein, the transcriptional activator protein (TrAP) activates late viral transcription [i.e. of the genes encoding the capsid protein (CP) gene and the nuclear shuttle protein (NSP)]. As reported by M. Boulton (Norwich), the gemini mastreviruses, like Maize streak virus and Bean yellow dwarf virus, use Rep for dsDNA cleavage and DNA polymerase sequestration and a separate protein (Rep-A) for cell cycle control and activation of late transcription.

Gronenborn also introduced the NMR-derived 3D structure of the aminoterminal domain of a geminivirus Rep protein (Gronenborn, 2004). It contains a typical central five-stranded $\beta$-sheet and a conserved helix, including the catalytically active tyrosine(s). This provides a rationale for the conservation of amino acid-sequence motifs in a large superfamily of replication initiator proteins of bacteriophages, circo-, and parvoviruses, and perhaps even VirD2 protein of the Ti-plasmid. 


\section{Movement}

The other nanovirus genes code for the capsid protein, for cell to cell movement and probably a NSP. There are also three genes of unknown function. S. Lazarowitz (Cornell) reported on the corresponding geminivirus proteins MPB (movement) and NSP (nuclear shuttle). NSP transports the viral DNA between nuclei and the cytoplasm. MPB traps the NSP-DNA complex within the cytoplasm and transports this to the plasma membrane and through the plasmodesmata into neighboring cells. To characterize the host cell transport and signaling pathways that are required for these transport events, S. Lazarowitz identified host proteins interacting with NSP and MPB (Figure 3). Arabidopsis nuclear shuttle interacting protein (AtNSI) is an acetyltransferase with the ability to oligomerize. It stably binds NSP and may regulate the nuclear export of the NSP:DNA complex. Lazarowitz hypothesizes that AtNSI, through its ability to acetylate the viral coat protein, inhibits coat protein interaction with viral ssDNA and allows NSP to compete for progeny genomes (see also Carvalho and Lazarowitz, 2004).

Lazarowitz also reported that Arabidopsis synaptogamin-like protein A (AtSYTA) interacts with geminivirus MPB and is necessary for MPB intercellular movement. It is one of five Arabidopsis proteins resembling mammalian synaptogamins. The latter are predominantly expressed in brain and are proposed to be involved in synaptic vesicle exocytosis and subsequent endocytosis. Interestingly the Tobacco mosaic virus (TMV) movement protein (MP) also interacts with AtSYTA suggesting that AtSYTA might be generally involved in the movement of viral and perhaps other RNAs. We are looking forward to verification of this interesting assumption. In line with these ideas, B Epel (Tel Aviv) reported on the incorporation of the TMV MP into the endoplasmic reticulum (ER) and hypothesized that the MP:ER complex aligns the viral RNA along the ER. Based on the connection of neighboring cells by the ER, he suggests that the cell-to-cell transport occurs via a type of pressure exerted by the accumulation of MP at the ER in the donor cell. It is of course tempting to speculate on the importance of vesicular trafficking in both human brain function and plant intercellular transport (see also Oparka, 2004). Do plants have thought and memory?

While geminivirus capsid protein is required for insect transmissibility, begomovirus cell-to-cell transport, and in many cases long range systemic movement, do not require capsid protein. It is therefore an open question as to why there is a strong selection for viral DNA size in cell-to-cell transport. R. Gilbertson (Davis) reported on his experiments on oversized Bean dwarf mosaic geminivirus-derived replicons expressing the green fluorescence reporter protein. These replicons were stable when replicated in single cells, but accumulated deletions that restored the original geminivirus genome size when moving from cell to cell (see also:

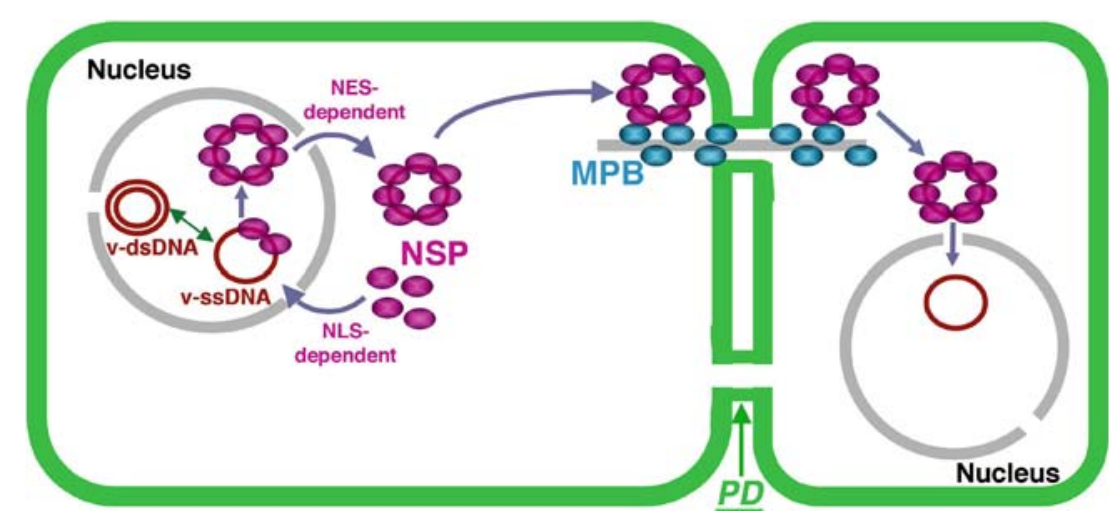

Figure 3. Model for begomovirus intra- and inter-cellular movement. The viral ssDNA genome replicates in the nucleus via dsDNA templates by a rolling circle mechanism. NSP, the viral-encoded NSP, binds progeny ssDNA genomes and transports these between the nucleus and cytoplasm. MPB, the viral cell-to-cell movement protein, traps NSP-genome complexes in the cytoplasm and directs these to and across the cell wall through modified plasmodesmata. In adjacent uninfected cells, NSP-genome complexes are released, and NSP targets the viral ssDNA to the nucleus to initiate new rounds of replication and infection. (Figure kindly provided by S. Lazarowitz). 
Gilbertson et al., 2003). Gilbertson suggested that combined properties of MPB and plasmodesmata contribute to the size limitations. Alternatively, MPB and/or NSP might form capsid-type structures of a specific size that limits the length of the packaged genome in a way similar to true viral capsids.

Nuclear import of both monopartite and bipartite geminivirus genomes in the original infection are likely mediated by capsid protein $(\mathrm{CP})$, while secondary infections of the nuclei of neighboring cells by bipartite geminiviruses are also mediated by NSP. A. Loyter (Rehovot) showed that both the monopartite Tomato yellow leaf curl virus $\mathrm{CP}$ and the bipartite Bean dwarf mosaic virus NSP have nuclear localization signals and bind specifically to importin $\alpha$. They can be imported into mammalian and plant nuclei both in their native form as well as when fused to fluorescently labeled BSA. Transport towards the nucleus might occur along microtubules (Salman et al., 2005).

\section{Silencing and resistance}

Viruses and hosts constantly fight each other on different battle fields. Maybe, the most important battle in plants occurs with the weapons of silencing and silencing suppression. During silencing the host recognizes dsRNA, or produces it from 'aberrant' or overproduced RNA by RNA dependent RNA polymerase. The dsRNA is then cleaved by dicer-like enzymes (DCL) into 21 to $24 \mathrm{nt}$ long small interfering (si)RNAs. From those a singlestranded derivative is incorporated into an RNA interference silencing complex (RISC), which scans RNAs for cognate sequences which are then cleaved (post-transcriptional gene silencing). Also cognate DNA sequences can be targeted leading to methylation of DNA and histone modification and thereby to transcriptional gene silencing.

Most studies concerning silencing of viruses have been performed with RNA viruses, by far the largest group of plant viruses. dsRNA being a byproduct of the RNA replication process of RNA viruses, is an obvious target of the host silencing machinery. However, these viruses counteract by producing silencing suppressors, which interfere with one or the other step of the silencing pathway.

Silencing suppression is an interesting phenomenon found to be connected to various proteins of RNA viruses and interfering with different steps of the silencing pathway. A much studied example is the helper component protein (HCPro) of potyviruses. For Zucchini yellow mosaic virus (ZYMV) A. Gal-On (Bet Dagan) reported that HCPro suppresses not only ZYMV silencing, but also breaks resistance to Cucumber mosaic virus (see also Wang et al., 2004). HCPro seems to act at the level of siRNA usage preventing disposal of the passenger siRNA. A point mutation of the critical HCPro FRNK motif to FINK is not inhibiting ZYMV accumulation, but reduces drastically the symptoms, perhaps by interfering with transcriptional control involved in innate host response.

At this conference it was reported that plants can also silence geminiviruses sometimes leading to recovery of the virus infected plants and that also geminiviruses respond to silencing by silencing suppression. This silencing process can be boosted by transiently transforming infected plants with dsRNA or DNA coding for ds (hairpin) RNA, as reported by T. Hohn (Basel) for Mung bean yellow mosaic virus. Stable transformation of cassava or Nicotiana benthamiana with African cassava mosaic virus (ACMV)-derived DNA cooling for hairpin RNA leads to early recovery of ACMV infection but not to total resistance. (Akbergenov et al., 2006 and references therein) In similar experiments reported by Y. Gafni (Bet Dagan), transient expression of cognate siRNA suppressed gene expression of the Tomato yellow leaf curl virus (TYLCV) coat protein gene and some tomato plants transgenic for a similar siRNA were resistant to the virus. T. Hohn also showed that all four plant $D C L$ genes are involved in siRNA production and that each of the DCL proteins is responsible for a specific size class ( $\mathrm{T}$ Blevins et al., personal communication).

Three groups showed that silencing suppression is not restricted to RNA viruses, but that also geminiviruses have silencing suppressors. Two genes of begomoviruses were found to act as suppressors, the transcription activator protein (TrAP or AC2; Trinks et al., 2005) and AC4 [reported by C. Fauquet (St Louis); see also Vanitherani et al., 2004]. According to C. Fauquet, some of the begomoviruses like African cassava mosaic virus (ACMV) use AC4 as an immediately acting but not lasting suppressor, while others like East African cassava mosaic virus (EACMV) use TrAP as a delayed but lasting suppressor. Interestingly, a most devastating 
disease results from double infection by these two viruses, probably due to concerted action of the two proteins. M. Boulton (Norwich) reported that both the Rep and RepA proteins of the monopartite mastrevirus Bean yellow dwarf virus, which function in replication, cell cycle activation and late virus gene activation function also as silencing suppressors as do the TrAPs of EACMV and Mungbean yellow mosaic virus (MYMV).

While for many viruses natural virus resistance markers are rare or not existing, there are exceptions. M. Lapidot and H. Czosnek (Bet Dagan) each reported on a tomato host resistant for TYLCV. In host TY-172 (M. Lapidot) the amount of viral ssDNA is much reduced suggesting that TY-172 interferes with TYLCV genome replication. By introgression of markers from wild tomato ( $L$. hirsutum) into the domesticated one (L. esculentum), lines tolerant $(\mathrm{lh} 902, \mathrm{R})$ and susceptible (Ie906,S) to TYLCV were developed (H. Czosnek). Genetic studies revealed that the tolerance is connected to a single locus and that it is accompanied by a decrease in abundance of MAP kinases upon inoculation with virus or application of other abiotic and biotic stresses.

\section{Why ssDNA?}

There was sufficient time during the intermissions to discuss research described above, but also other questions were subjects of intense dispute, as for instance that on the stability and survival of ssDNA in eukaryotes. ssDNA as such does not exist in eukaryotic organisms, apart from necessary and short-lived stretches during DNA replication and DNA repair. Hosts must have 'learned' to recognize ssDNA as parasitic and developed strategies for its elimination, much as hosts have 'learned' to recognize and eliminate virus- and transposon-derived dsRNA by silencing. However, also the plant pathogenic ssDNAs are coated with Ti-plasmid- and virus-coded proteins, respectively. These proteins protect the DNA from degradation and guide their intra- and inter-cellular movement. In addition, they may help the pathogen to avoid an SOS-like response reaction as known for prokaryotic organisms (reviewed by Michel, 2005).

Why then have ssDNA parasites survived and what is the advantage of this form of genome? One advantage (shared with ssRNA) is the adaptability to new conditions, hosts and environments due to the high recombination- and mutation rate of single-stranded nucleic acids. On the other hand, once transformed into dsDNA, their genetic information is stably conserved. Another reason for single-strandedness might be the limiting space that viral capsids provide. Single-stranded genomes have half the molecular weight than double-strand ones and in addition, might be more flexible and compressible when packaged. The reduced molecular weight and increased flexibility will also help ssDNA to pass barriers, such as the secretory machinery formed by the Agrobacterium, the nuclear pore used by both agrobacterial T-DNA and viral genomes and the plasmodesmata, gateways for viral spread. Also intracellular movement along cytoskeletal elements and via cytoplasmic vesicles could be enhanced.

As in most successful meetings more questions arose than were finally answered. However, every increment in knowledge on the life cycle of these intriguing parasites will not only advance basic plant and microbial science, where T-DNA and geminiviruses are our love and toys, but will also get us closer to resistance breeding; in agriculture, after all, these interesting bugs are our enemies. It is to be hoped, therefore, that conferences, such as the Eilat-meeting, will be repeated.

\section{Acknowledgements}

We thank all participants of the conference who provided figures and unpublished information. Special thanks go to S. Gelvin, B. Gronenborn and S. Lazarowitz for helpful comments on this manuscript and to M. Duckely, V. Citovsky and S. Lazarowitz for providing the illustrations. This workshop was supported by BARD Award No. W-68-05.

\section{References}

Abu-Arish, A., Frenkiel-Krispin, D., Fricke, T., Tzfira, T., Citovsky, V., Wolf, S.G. and Elbaum, M. 2004. Threedimensional reconstruction of Agrobacterium VirE2 protein with single-stranded DNA. J. Biol. Chem. 279: 25359-25363.

Akbergenov, R., Si-Ammour, A., Blevins, T., Amin, I., Kutter, C., Vanderschuren, H., Zhang, P., Gruissem, W., Meins, F. 
Jr., Hohn, T., Pooggin, M.M. 2006. Molecular characterization of geminivirus-derived small RNAs in different plant species. Nucleic Acides Res 34: 462-471.

Carvalho, M.F. and Lazarowitz, S.G. 2004. Interaction of the movement protein NSP and the Arabidopsis acetyltransferase AtNSI is necessary for cabbage leaf curl geminivirus infection and pathogenicity. J. Virol. 78: 11161-11171.

Cascales, E. and Christie, P.J. 2004. Definition of a bacterial type IV secretion pathway for a DNA substrate. Science 304: $1170-1173$.

Citovsky, V., Zupan, J., Warnick, D. and Zambryski, P. 1992. Nuclear localization of Agrobacterium VirE2 protein in plant cells. Science 256: 1802-1805.

Ditt, R.F., Nester, E. and Comai, L. 2005. The plant cell defense and Agrobacterium tumefaciens. FEMS Microbiol. Lett. 247: 207-213.

Dumas, F., Duckely, M., Pelczar, P., Van Gelder, P. and Hohn, B. 2001. An Agrobacterium VirE2 channel for transferredDNA transport into plant cells. Proc. Natl. Acad. Sci. USA 98: 485-490.

Gelvin, S.B. 2003. Agrobacterium-mediated plant transformation: the biology behind the 'gene-jockeying' tool. Microbiol. Mol. Biol. Rev. 67: 16-37.

Gilbertson, R.L., Sudarshana, M., Jiang, H., Rojas, M.R. and Lucas, W.J. 2003. Limitations on geminivirus genome size imposed by plasmodesmata and virus-encoded movement protein: insights into DNA trafficking. Plant Cell 15: 25782591.

Gronenborn, B. 2004. Nanoviruses: genome organisation and protein function. Vet. Microbiol. 98: 103-109.

Li, J., Vaidya, M., White, C., Vainstein, A., Citovsky, V. and Tzfira, T. 2005. Involvement of KU80 in T-DNA integration in plant cells, Proc. Natl. Acad. Sci. USA 102: 19231-19236.
Loyter, A., Rosenbluh, J., Zakai, N., Li, J., Kozlovsky, S.V., Tzfira, T. and Citovsky, V. 2005. The plant VirE2 interacting protein 1, a molecular link between the Agrobacterium T-complex and the host cell chromatin?. Plant Physiol. 138: 1318-1321.

Michel, B. 2005. After 30 years of study, the bacterial SOS response still surprises us. PLoS Biol. 3: e255.

Oparka, K.J. 2004. Getting the message across: how do plant cells exchange macromolecular complexes? Trends Plant Sci. 9: 33-41.

Rosen, R., Becher, D., Büttner, K., Biran, D., Hecker, M. and Ron, E.Z. 2004. Highly phosphorylated bacterial proteins. Proteomics 4: 3068-3077.

Salman, H., Abu-Arish, A., Oliel, S., Loyter, A., Klafter, J., Granek, R. and Elbaum, M. 2005. Nuclear localization signal peptides induce molecular delivery along microtubules. Biophys. J. 89: 2134-2145.

Trinks, D., Rajeswaran, R., Shivaprasad, P.V., Akbergenov, R., Oakeley, E.J., Veluthambi, K., Hohn, T. and Pooggin, M.M. 2005. Suppression of RNA silencing by a geminivirus nuclear protein, $\mathrm{AC} 2$, correlates with transactivation of host genes. J. Virol. 79: 2517-2527.

Vanitharani, R., Chellappan, P., Pita, J.S. and Fauquet, C.M. 2004. Differential roles of AC2 and AC4 of cassava geminiviruses in mediating synergism and suppression of posttranscriptional gene silencing. J. Virol. 78: 9487-9498.

Wang, Y., Lee, K.C., Gaba, V., Wong, S.M., Palukaitis, P. and Gal-On, A. 2004. Breakage of resistance to cucumber mosaic virus by co-infection with zucchini yellow mosaic virus: enhancement of CMV accumulation independent of symptom expression. Arch. Virol. 149: 379-396. 